\title{
Lung ultrasound for early diagnosis and severity assessment of pneumonia in patients with coronavirus disease 2019
}

Young-Jae Cho, ${ }^{1,}$, Kyoung-Ho Song ${ }^{2,}$, Yunghee Lee ${ }^{1}$, Joo Heung Yoon ${ }^{3}$, Ji Young Park ${ }^{4,5}$, Jongtak Jung ${ }^{2}$, Sung Yoon Lim ${ }^{1}$, Hyunju Lee, Ho Il Yoon ${ }^{1}$, Kyoung Un Park ${ }^{6}$, Hong Bin Kim², and Eu Suk Kim²

Divisions of ${ }^{1}$ Pulmonary and Critical Care Medicine and ${ }^{2}$ Infectious Diseases, Department of Internal Medicine, Seoul National University Bundang Hospital, Seongnam, Korea; ${ }^{3}$ Division of Pulmonary, Allergy, and Critical Care Medicine, Department of Medicine, University of Pittsburgh Medical Center, Pittsburgh, PA, USA; ${ }^{4}$ Department of Paediatrics, ChungAng University Hospital, Seoul; Departments of ${ }^{5}$ Paediatrics and

${ }^{6}$ Laboratory Medicine, Seoul National University Bundang Hospital,

Seongnam, Korea

Received: April 26, 2020

Revised : May 12, 2020

Accepted: May 19, 2020

\section{Correspondence to}

Eu Suk Kim, M.D.

Division of Infectious Diseases,

Department of Internal

Medicine, Seoul National

University Bundang Hospital, 82

Gumi-ro 173beon-gil, Bundang-

gu, Seongnam 13620, Korea

Tel: +82-31-787-7062

Fax: $+82-31-787-4052$

E-mail: eskim@snubh.org

https://orcid.org/0000-0001-

7132-0157

*These authors contributed equally to this work.
Background/Aims: Current evidence supports lung ultrasound as a point-ofcare alternative diagnostic tool for various respiratory diseases. We sought to determine the utility of lung ultrasound for early detection of pneumonia and for assessment of respiratory failure among patients with coronavirus disease 2019 (COVID-19).

Methods: Six patients with confirmed COVID-19 by reverse transcription-polymerase chain reaction were enrolled. All had undergone chest X-ray and chest computed tomography (CT) on the day of admission and underwent multiple point-of-care lung ultrasound scans over the course of their hospitalization.

Results: Lung ultrasound detected early abnormal findings of representative B-lines in a patient with a normal chest X-ray, corresponding to ground-glass opacities on the chest CT scan. The ultrasound findings improved as her clinical condition improved and her viral load decreased. In another minimally symptomatic patient without significant chest X-ray findings, the ultrasound showed B-lines, an early sign of pneumonia before abnormalities were detected on the chest CT scan. In two critically ill patients, ultrasound was performed to assess for evaluation of disease severity. In both patients, the clinicians conducted emergency rapid sequence intubation based on the ultrasound findings without awaiting the laboratory results and radiological reports. In two children, ultrasound was used to assess the improvement in their pneumonia, thus avoiding further imaging tests such as chest CT.

Conclusions: Lung ultrasound is feasible and useful as a rapid, sensitive, and affordable point-of-care screening tool to detect pneumonia and assess the severity of respiratory failure in patients hospitalized with COVID-19.

Keywords: Ultrasonography; Pneumonia; Respiratory insufficiency; Severe acute respiratory syndrome coronavirus 2; COVID-19

\section{INTRODUCTION}

The world is currently experiencing a novel coronavirus disease 2019 (COVID-19) pandemic [1]. In South Korea, there have been multiple confirmed cases related to local outbreak clusters, but there are no rapid, sensitive, affordable screening tools available to assess the presence of pneumonia, disease severity, or risk of respiratory fail- 

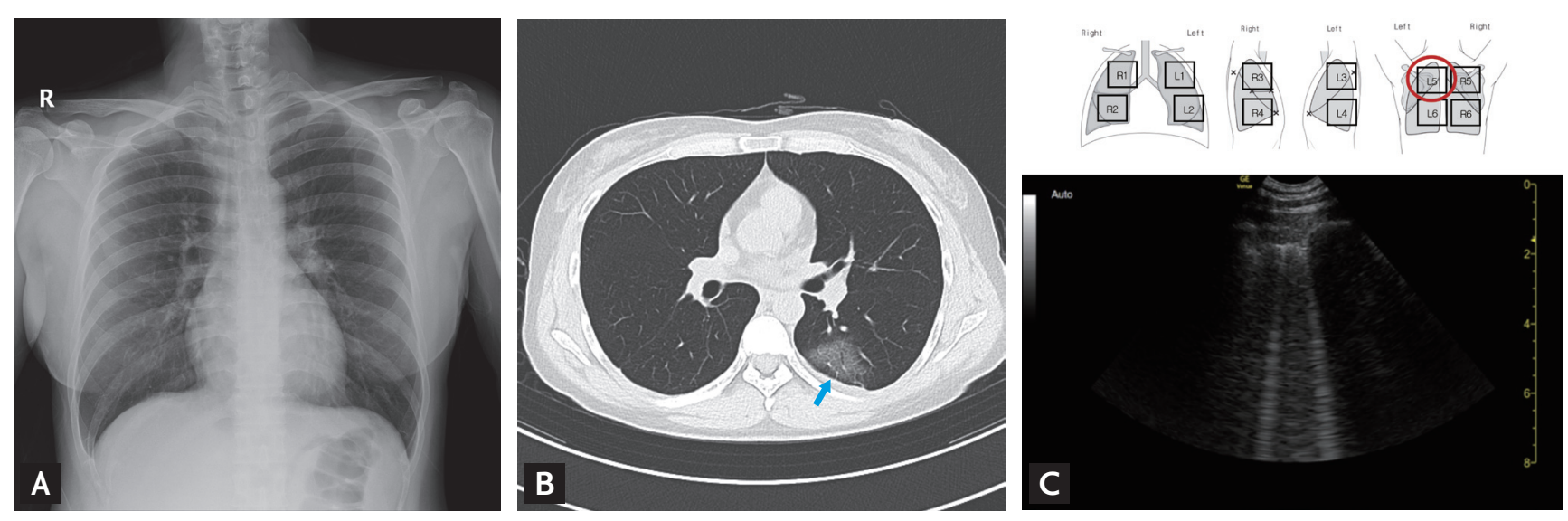

Figure 1. Case 1: Initial chest X-ray, computed tomography, and lung ultrasound findings in L5; (A) the chest X-ray was normal, (B) the chest computed tomography showed ground-glass opacity (blue arrow) in the posterior left upper lung, and (C) the lung ultrasound showed typical B-lines in L5.

ure. Chest computed tomography (CT) is a useful method for diagnosing COVID-19 [2]. However, the highly transmittable nature of COVID-19 through droplets or fomites, and the risk associated with radiation exposure, has hindered routine use of traditional diagnostic modalities such as computed tomography (CT) [3-5].

Lung ultrasound (LUS) is increasingly performed at the bedside for clinical assessment of the patients with acute respiratory failure [6]. The normal lung surface associate lung sliding with horizontal repetitions of the pleural line, called A-lines. 'B-lines' indicate loss of lung aeration. The Bedside Lung Ultrasound in an Emergency (BLUE) protocol is a fast protocol ( $<3$ minutes), which combines these signs, resulting in several aeration patterns: moderate loss of lung aeration ( $\mathrm{B} 1$ lines); severe loss of lung aeration (B2 lines); pulmonary consolidation (C).

Herein, we describe some of the key LUS findings in six patients with confirmed COVID-19 infection with normal to minimal radiographic changes; and COVID-19 pneumonia along their clinical course, to demonstrate the feasibility and utility of the LUS in early detection of pneumonia and clinical guidance on the management of the acute respiratory failure.

\section{METHODS}

\section{Cases and specimen collection}

We retrospectively reviewed the records of six consecutive patients with confirmed COVID-19 who underwent serial LUS. All patients had routine clinical admission evaluation with repeated reverse transcription-polymerase chain reaction (RT-PCR) tests for severe acute respiratory syndrome coronavirus 2 (SARS-CoV-2) in samples obtained from their upper or lower respiratory tract during their hospitalisation. The patients' treatment differed according to the decision of the primary attending physician, who is an infectious disease specialist. All LUS were conducted by one independent pulmonary and critical care specialist using a point-of-care ultrasound.

\section{Lung ultrasound}

For LUS examination (Supplementary Fig. 1), an 8C-RS microconvex probe (8 MHz, Venue, GE Healthcare, Milwaukee, WI, USA), which directly applied to the intercostal space was used. The use of bedside ultrasound by the International Consensus Conference was not considered in the report [7]. Patients were examined in sitting or semi-recumbent position, with the probe applied perpendicularly to the chest surface, as the patient's clinical condition allowed [8].

Six areas per each hemithorax were examined, as recommended [6,9]. At least two central intercostal spaces per area were investigated with longitudinal or transversal scans. First, the scan was done to correctly identify the pleura; a video clip at least one respiratory cycle length was stored for offline analysis. Then, the following data were recorded for each scan: the length of pleural line, presence of A-lines, number and coalescence of B-lines, subpleural echo-poor regions (subpleural con- 
solidations), and tissue-like patterns (consolidations). To quantify B-lines, we employed a machine learning system embedded in the Venue device, which included "auto-gain" and "auto B-lines" functions [10]. The "auto B-lines" function automatically distinguishes between real B-lines and all other artefacts [11].

The Lung Review screen provided the overall LUS score, by adding the scores from 12 segments. Each B-line was counted and the count per zone was given as one of the following numbers: $0,1,2,3,4$, or $\geq 5$. The score for each segment was defined as follows: counts $\leq 2$ were given a score of $\mathrm{O}$; counts of 3 or 4 were given a score of 1 (B1lines); counts $\geq 5$ were given a score of 2 (B2-lines); and if consolidation was present a score of $3(\mathrm{C})$ was given (The finding of consolidation was not automatically recorded and had to be entered manually). The "Auto B-lines" tool highlighted B-lines while scanning, and at the end of the sweep the line tool jumped image to the frame with the most B-lines. Some of these key features included the brightness of the $\mathrm{B}$-lines relative to its surroundings, the depth of B-lines (whether the B-lines extended to the bottom of the image), and the deletion of A-lines.

\section{Ethics}

The study protocol was approved by Institutional Review Board of Seoul National University, Bundang Hospital(B2004-607-107). The Institutional Review Board waived the need for informed consent because the study was based on a retrospective analysis of electronic medical records.

\section{RESULTS}

The records of six patients (four adults and two children) were analysed retrospectively. Five patients showed positive RT-PCR for SARS-CoV-2 in upper and lower respiratory tract specimens, and one 15-month-old girl was only positive in the upper respiratory tract as a lower respiratory tract specimen was not able to be obtained. The clinical characteristics of the enrolled patients are shown in Table 1. Of the four adults, the first was a 40-year-old woman who had a sore throat and a fever which had started 5 days previously, and the second was a 6o-year-old man with a cough. The two critically ill patients were a 62-year-old woman and an 85-year-old man, both with dyspnoea, and transferred from other hospital for critical care. Of the two children, the first was a 10-year-old girl who had a small amount of sputum for 3 days. The second was a 15-month-old girl who had tested positive for SARS-CoV-2 but had no respiratory symptoms. Of the four adults, LUS use in two cases led to the with early detection of pneumonia, and two cases with severity assessment for acute respiratory failure. LUS was followed by the results of chest X-ray (CXR) and chest $\mathrm{CT}$ results focusing on abnormal findings in the index cases, and then followed by LUS prospectively.

\section{Use of lung ultrasound for early detection of pneumonia}

\section{Case 1}

A 40-year-old woman was referred and admitted following a positive SARS-CoV-2 test at a community health screening, which she attended 5 days after the onset of a sore throat and fever. She had had contact with a family member with a confirmed COVID-19. She did not have symptoms of a lower respiratory tract infection. The CXR performed on the day of visit was normal (Fig. 1A), but her chest CT (Fig. 1B) performed on Day 2 showed ground-glass opacities (GGOs) in the left upper lung field. A LUS was performed and showed typical B-lines (Fig. 1C, Video 1; https://www.kjim.org), which findings were consistent with the CT abnormalities. Also, typical A-lines (normal aeration) (Fig. 2, Video 2; https://www. kjim.org/) were observed on the opposite side in the
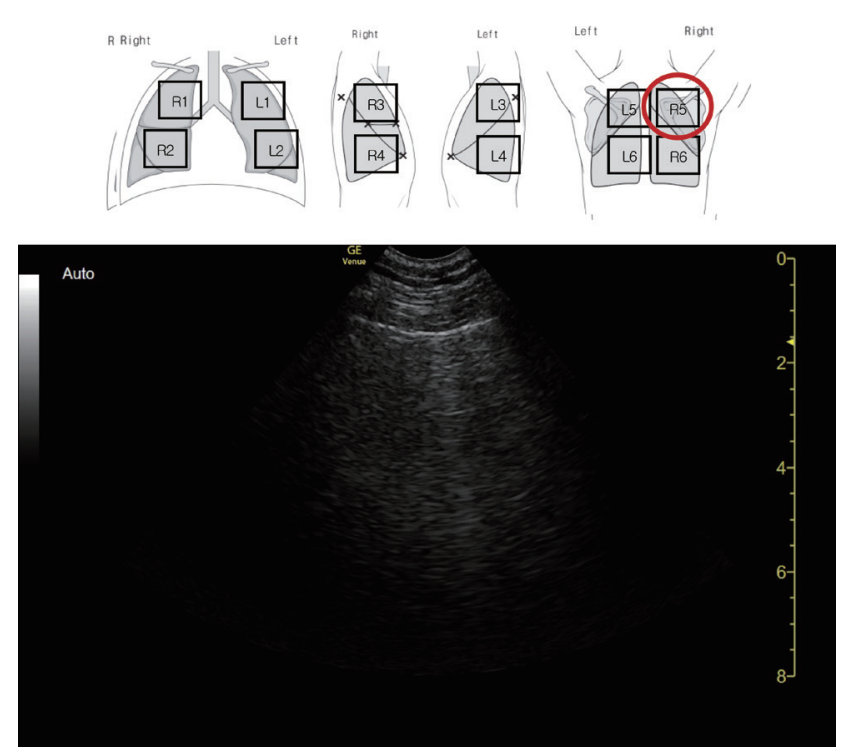

Figure 2. Case 1: Normal lung ultrasound findings with A-lines in R5 on same day of Fig. 1. 
Table 1. Characteristics of enrolled patients

\begin{tabular}{|c|c|c|c|c|c|c|}
\hline Characteristic & 1 & 2 & 3 & 4 & 5 & 6 \\
\hline $\operatorname{Sex}$ & $\mathrm{F}$ & $\mathrm{M}$ & $\mathrm{F}$ & M & $\mathrm{F}$ & $\mathrm{F}$ \\
\hline Age, yr & 40 & 60 & 62 & 85 & 10 & 16 mon \\
\hline \multicolumn{7}{|l|}{ Symptoms } \\
\hline Fever $\left(>37.5^{\circ} \mathrm{C}\right)$ & - & + & + & - & - & - \\
\hline Rhinorrhea/nasal obstruction & - & - & - & - & + & - \\
\hline Sore throat & + & - & - & - & - & - \\
\hline Cough & - & - & - & - & - & - \\
\hline Sputum & - & - & - & - & + & - \\
\hline Dyspnea & - & - & + & + & - & - \\
\hline Fatigue & - & - & - & - & - & - \\
\hline Nausea/Vomiting & - & - & - & - & - & - \\
\hline Diarrhea & - & - & - & - & - & - \\
\hline Others & Back pain & Myalgia & - & - & - & - \\
\hline \multicolumn{7}{|l|}{ Clinical laboratory results } \\
\hline $\mathrm{WBC}, / \mu \mathrm{L}$ & 4,450 & 7,610 & 3,620 & 9,110 & 4,080 & 6,800 \\
\hline Neutrophils, $/ \mu \mathrm{L}$ & 2,003 & 5,860 & 2,911 & 8,472 & 2,228 & 1,809 \\
\hline Lymphocytes, / $\mu \mathrm{L}$ & 2,052 & 609 & 521 & 437 & 1,522 & 3,522 \\
\hline $\mathrm{PLT}, / \mu \mathrm{L}$ & 169,000 & 197,000 & 84,000 & 93,000 & 251,000 & 235,000 \\
\hline Hemoglobin, g/dL & 12.7 & $15 \cdot 6$ & 10.3 & 11.6 & $13 \cdot 5$ & 12.1 \\
\hline Hematocrit, \% & 38.0 & $45 \cdot 3$ & 31.1 & 33.8 & $39 \cdot 9$ & $37 \cdot 5$ \\
\hline Sodium, mmol/L & 140 & 138 & 142 & 140 & 140 & 137 \\
\hline Potassium, mmol/L & 3.8 & 4.0 & $3 \cdot 3$ & 4.1 & 3.8 & $4 \cdot 3$ \\
\hline Chloride, mmol/L & 104 & 104 & 111 & 109 & 108 & 107 \\
\hline Calcium, mg/dL & 8.6 & 9.0 & $7 \cdot 5$ & 8.4 & 9.2 & $9 \cdot 7$ \\
\hline $\mathrm{CO}_{2}, \mathrm{mmHg}$ & $\mathrm{NC}$ & $37 \cdot 5$ & 40.3 & 46.1 & 25.0 & $\mathrm{NC}$ \\
\hline Glucose, mg/dL & 85 & 145 & 105 & 112 & 106 & 102 \\
\hline $\mathrm{BUN}, \mathrm{mg} / \mathrm{dL}$ & 9 & 9 & 12 & 37 & 15 & 15 \\
\hline Creatinine, mg/dL & 0.7 & 0.8 & 0.69 & 1.12 & 0.45 & 0.35 \\
\hline Total protein, g/dL & $7 \cdot 6$ & 7.1 & 6.2 & 6.8 & 7.0 & $7 \cdot 0$ \\
\hline Albumin, g/dL & 4.2 & $4 \cdot 5$ & 3.0 & $3 \cdot 3$ & $4 \cdot 5$ & 4.7 \\
\hline $\mathrm{TB}, \mathrm{mg} / \mathrm{dL}$ & 0.6 & 0.5 & 0.4 & 2.8 & 0.4 & 0.3 \\
\hline Procalcitonin, ng/mL & $\mathrm{NC}$ & $\mathrm{NC}$ & 0.14 & 30 & $\mathrm{NC}$ & $\mathrm{NC}$ \\
\hline CRP, mg/dL & $<0.4$ & $<0.4$ & 8.65 & $\mathrm{NC}$ & $<0.4$ & $<0.4$ \\
\hline ALT, U/L & 36 & 16 & 23 & 11 & $<9$ & 21 \\
\hline AST, U/L & 36 & 24 & 60 & 88 & 20 & 55 \\
\hline $\mathrm{ALP}, \mathrm{U} / \mathrm{L}$ & 53 & 73 & 84 & 134 & 189 & 287 \\
\hline Fibrinogen, mg/dL & 331 & 296 & 401 & 540 & $\mathrm{NC}$ & $\mathrm{NC}$ \\
\hline PT, sec & 12.7 & 12.4 & 12.7 & 13.7 & $\mathrm{NC}$ & $\mathrm{NC}$ \\
\hline INR & 0.97 & 0.94 & 0.97 & 1.06 & $\mathrm{NC}$ & $\mathrm{NC}$ \\
\hline
\end{tabular}

WBC, white-cell count; PLT, platelet count; $\mathrm{CO}_{2}$, carbon dioxide; NC, not checked; BUN, blood urea nitrogen; TB, total bilirubin; CRP, C-reactive protein; ALT, alanine aminotransferase; AST, aspartate aminotransferase; ALP, alkaline phosphatase; PT, prothrombin time; INR, international normalized ratio. 

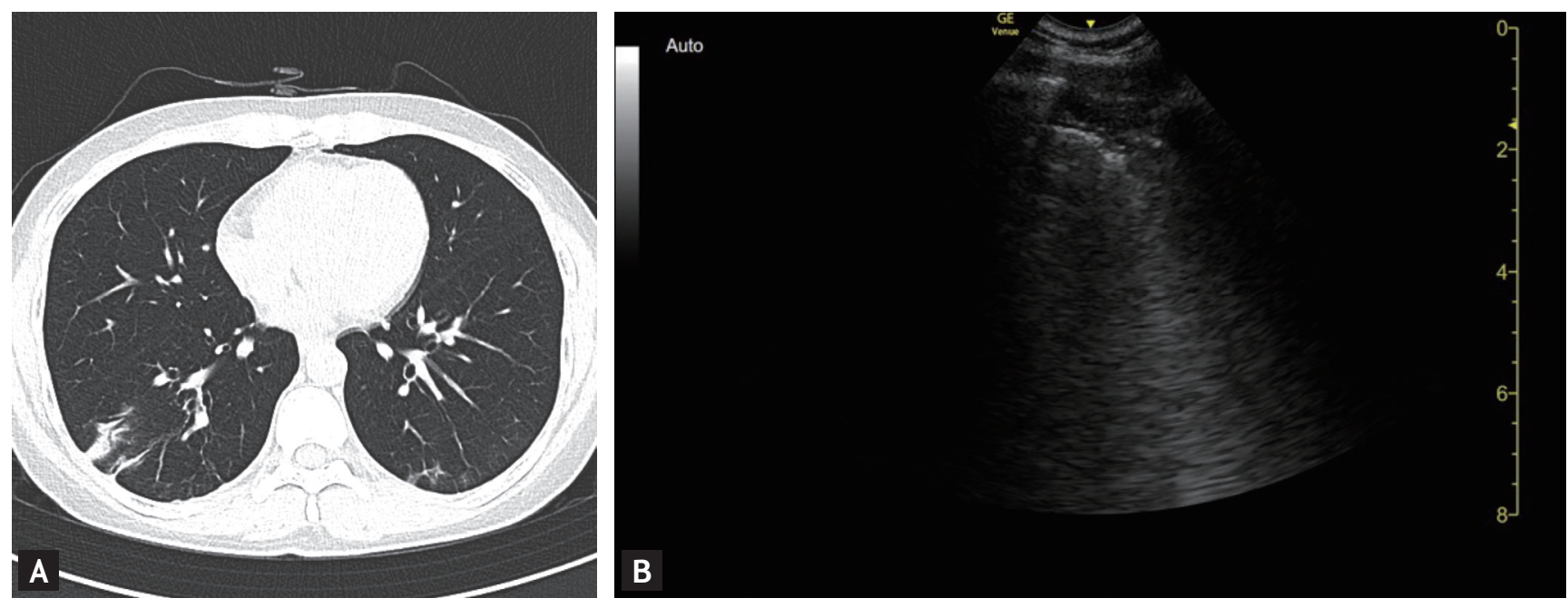

Figure 3. Case 1: The computed tomography (A) and lung ultrasound (B) findings of subpleural consolidation in the right lower lung field.

right upper lung field, which corresponded with normal CT findings on the right. She developed dry cough and fever on Day 5 and began treatment with lopinavir/ritonavir. Contrary to near minimal changes of serial CXR (Supplementary Fig. 2A), second chest CT performed on the day of starting treatment (Day 6) also showed more pronounced GGOs (Supplementary Fig. $2 \mathrm{~B}$ ), where the B-lines were already increased in the same area as the LUS performed on Day 4, 1 day before worsening symptoms (Supplementary Fig. 2C, Supplementary Video 1; https://www.kjim.org). Her symptoms improved, and the B-lines gradually decreased in the LUSs performed on Day 9 and Day 16 after admission. Finally, the B-lines disappeared and were replaced by A-lines. The GGOs became less marked in the chest CT conducted on Day 14. Beside left upper lung field, additional consolidation was seen at the right lower lung field in the CT performed on Day 14 (Fig. 3A), and the LUS revealed subpleural consolidation accompanied by B-lines on Day 16 (Fig. 3B, Video 3; https://www.kjim.org).

\section{Case 2}

A 6o-year-old man was admitted to the hospital following a positive SARS-CoV-2 test, with a 1-day history of cough, chills, and myalgia. He had been exposed to a colleague with SARS-CoV-2 infection, who had experienced similar symptoms 5 days before the onset of his symptoms. At the time of hospitalisation, he did not complain of any symptoms except for feeling feverish, and findings from the CXR and CT performed on the day of admission were normal. While he became febrile, a combination of lopinavir/ritonavir was started on Day 5. On Day 8, a repeat chest CT (Supplementary Fig. 3A) and CXR (Supplementary Fig. 3B) showed relatively dense GGOs in the right upper lung field.

A LUS performed on Day 9 showed B-lines (Supplementary Fig. 3C, Supplementary Video 2; https://www. kjim.org) in the area where the GGOs had been seen on the CT performed on the previous day. The opposite left side lung field showed GGOs in CXR on Day 13 (Supplementary Fig. 4A) and chest CT on Day 15 (Supplementary Fig. $4 \mathrm{~B}$ ); however, the LUS already showed B-lines (Supplementary Fig. 4C, Supplementary Video 3; https:// www.kjim.org) at same field on Day 9. The LUS detected subtle abnormalities before the CXR or CT with clinical deterioration.

\section{Use of lung ultrasound for severity assessment for acute respiratory failure}

\section{Case 3}

An 85-year-old man was transferred from another hospital with ongoing respiratory problems. He had been diagnosed with confirmed COVID-19 at the other hospital, 6 days before being transferred to our hospital. Immediately before transfer, the patient had rapid, shallowing 


\section{KJIM}
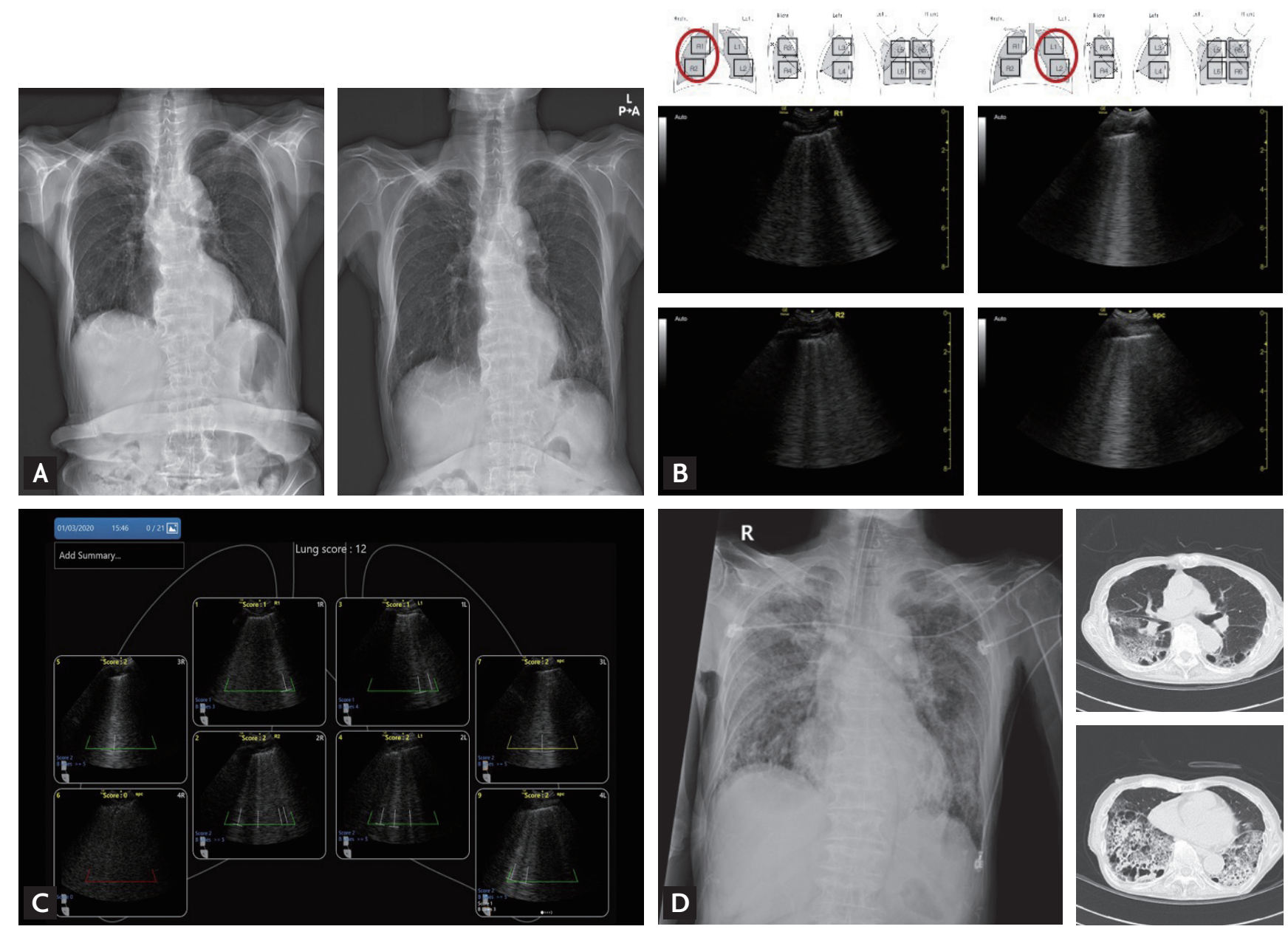

Figure 4. Case 3: The chest X-rays taken 5 days, and 1 day (A) before being transferred to our hospital; The lung ultrasound showed both B-lines in both anterior lung field (B) with aeration score 12 (C); the chest X-ray (left panel) in intubated state and chest computed tomography scan (right panel) showing bilateral diffuse ground glass opacities and consolidations (D), which suggested acute respiratory distress syndrome.

breathing despite oxygen supplementation. Copies of CXRs taken 5 days, and 1 day before being transferred to our hospital are shown in Fig. 4A. LUS was immediately performed to assess the extent of lung infiltration. The LUS showed multiple B-lines in the entire anterior bilateral upper and lower lung fields (Fig. 4B), and the lung aeration score according to the automated protocol was 12 (Fig. 4C). Based on these findings, we immediately decided to initiate mechanical ventilation with rapid sequence intubation. A post-intubation CXR and chest CT showed typical findings of adult respiratory distress syndrome (ARDS) (Fig. 4D).

\section{Case 4}

A 62-year-old woman was transferred from another hos- pital after being diagnosed with COVID-19. She had been admitted to the other hospital after 8 days of fever, when she developed respiratory distress occurred during isolation. At the time of her transfer to our hospital she was being treated with empirical antibiotics and oseltamivir and was receiving $4 \mathrm{~L} / \mathrm{min}$ of supplemental oxygen via nasal prongs. Her CXR and CT findings at the previous hospital are shown in Supplementary Fig. 5A. The LUS was performed with the patient in the supine position. Multiple B-lines were observed in the anterior right upper lung field, and both anterior lower lung fields, but not the anterior left upper lung field. Her lung aeration score was 3 points (Supplementary Fig. 5 B), based on the LUS findings in both anterior areas. Although lopinavir/ ritonavir was started immediately after transfer to our 
Table 2. The time course of disease progression in case 3

\begin{tabular}{|c|c|c|c|c|c|}
\hline Hospital course & Day 1 & Day 4 & Day 8 & Day 16 & Day 18 \\
\hline $\mathrm{PaO}_{2}(\mathrm{mmHg}) / \mathrm{FiO}_{2}$ & $68.5 / 0.36$ & $95 \cdot 4 / 0.5$ & $79.8 / 0.6$ & $56.8^{\mathrm{a}} / 0.7$ & $118.6 / 0.4$ \\
\hline $\mathrm{PaO}_{2} / \mathrm{FiO}_{2}$ ratio & 190 & 190.8 & 133 & $81.1^{\mathrm{a}}$ & 296.5 \\
\hline Lung score & 3 & 5 & 4 & $\mathrm{NC}$ & 13 \\
\hline Steroid & - & + & + & + & + \\
\hline Intubation & - & + & + & + & + \\
\hline Inhaled nitric oxide & - & - & + & + & - \\
\hline ECMO & - & - & - & + & + \\
\hline
\end{tabular}

$\mathrm{PaO}_{2}$, partial pressure of oxygen; $\mathrm{FiO}_{2}$, fraction of inspired oxygen; NC, not checked; ECMO, extracorporeal membrane oxygenation.

${ }^{\mathrm{a} C h e c k e d ~ b e f o r e ~ E C M O . ~}$
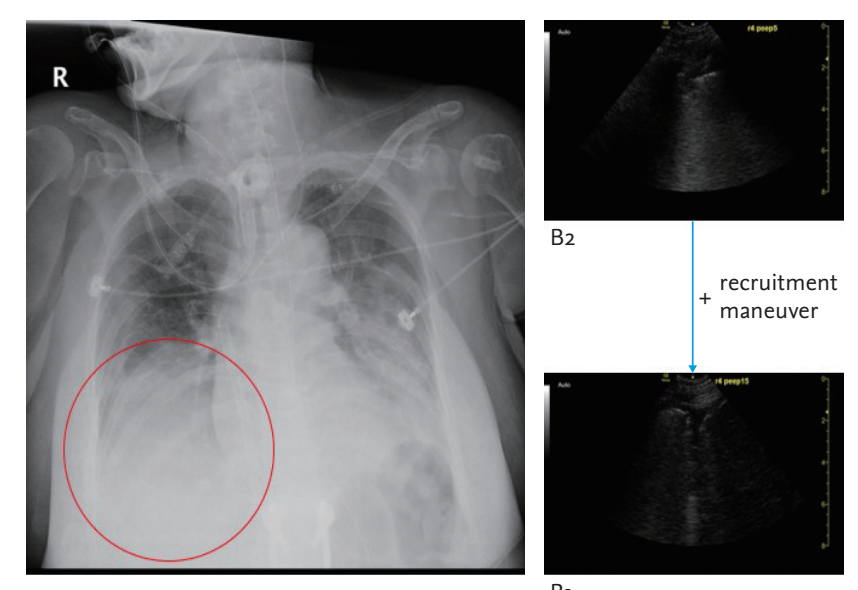

B1

Figure 5. Case 4: A recruitment maneuver resulted in improved findings in $\mathrm{R}_{4}$ on the lung ultrasound from B2-lines to Br-lines.

hospital, she was intubated on Day 3 after her respiratory symptoms became more severe. LUS performed on Day 4 showed a worsening of her pneumonia, with the lung aeration score of 5 points (Supplementary Fig. ${ }_{5} \mathrm{C}$ ). After mechanical ventilation, the patient's CXR and LUS aeration score improved from 5 to 4 , but her hypoxia remained severe (ratio of arterial oxygen partial pressure to fractional inspired oxygen [P/F ratio] 133 on Day 8). As a rescue therapy for ARDS, methylprednisolone (1 mg/ $\mathrm{kg}$ intravenously) and inhaled nitric oxide were administered.

Despite these interventions, the patient continued to deteriorate, and veno-venous extracorporeal membrane oxygenation (ECMO) support was started on Day 16. Her CXR showed worsening of her pneumonia, and a LUS performed on Day 18 (2 days after ECMO application) showed a lung aeration score of 13 points (Supplementary Fig. $5 \mathrm{D}$ ), which was worse than it had been on Day 3 (the day of intubation). (Table 2) The patient was subsequently diagnosed with hospital-acquired pneumonia caused by carbapenem-resistant Acinetobacter baumannii cultured from a respiratory sample.

Under the ECMO support, LUS was performed before and after an alveolar recruitment manoeuvre to assess proper positive end-expiratory pressure (PEEP) on the ventilator. The LUS performed after the recruitment maneuver revealed improved aeration of the right lower lung field and lowering of the diaphragm level followed by lung expansion (Fig. 5). Based on that findings on the LUS, we were able to decide on the optimal PEEP settings and performed a follow-up CXR to confirm improved lung recruitment.

\section{Use of lung ultrasound in children}

\section{Case 5}

A 10-year-old girl had confirmed SARS-CoV-2 infection after being exposed to a family member with COVID-19 and was hospitalised for evaluation and monitoring of her symptoms. The initial CXR after admission was normal. However, her chest CT scan on Day 2 showed focal consolidation with multiple GGOs in the right lower lung field (Supplementary Fig. 6). As in Case 1, the LUS (performed on Day 3), showed typical B-lines (consolidations and GGOs) in the right lung field (Fig. 6A), and contralateral A-lines (normal aeration) in the left lower lung field. She developed a small amount of sputum 

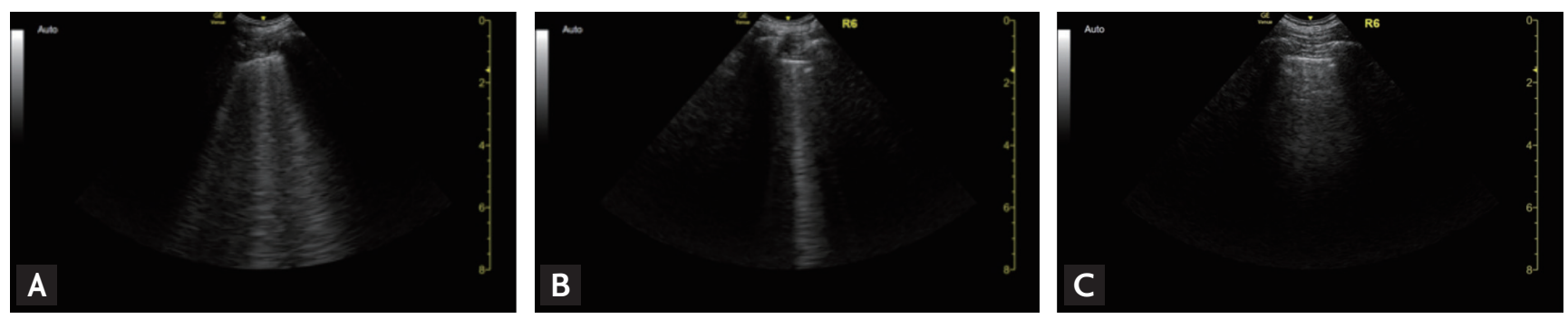

Figure 6. Case 5: Gradual improvement of lung ultrasound in R6 from B2-lines (A) through B1-lines (B), then to A-lines (C).

but was not administered medication for pneumonia because her condition was stable. Follow-up CXRs and LUS were performed 4 and 6 days, respectively, after the first LUS. As her symptoms improved, LUS showed evolution from B2-lines to B1-lines and then A-lines, indicating progressive improvement in her lung aeration (Fig. 6B and 6C, Video 4; https://www.kjim.org). Since there was no evidence of worsening symptoms during the hospitalisation, the patient was transferred to the step-down facility. The final lung aeration score was o and her symptoms also disappeared at that time.

\section{Case 6}

A 15-month-old girl was hospitalised with SARS-CoV-2 infection after contracting infection from a member of her family. She had no symptoms of COVID-19 and was clinically stable. The initial CXR also showed normal findings (Supplementary Fig. 7A). To further rule out the presence of pneumonia, LUS and CXR was carefully performed on her back area at the bedside with the support of her father on Day 2. The LUS showed A-lines throughout her lungs, and no B-lines (Supplementary Fig. $7 \mathrm{~B}$ ). Chest $\mathrm{CT}$ was not performed because of her stable clinical condition and normal CXR and LUS findings. The CXR (Supplementary Fig. $7 \mathrm{C}$ ) and LUS were repeated on Day 4 and remained normal. The patient was later discharged without requiring treatment.

\section{DISCUSSION}

This is the first LUS case series performed for COVID-19 patients in South Korea. In Cases 1 and 2, LUS showed important early findings of pneumonia, which corresponded to the findings on chest CT, while CXR did not reveal abnormalities. LUS is an especially useful tool for COVID-19 patients because performing conventional auscultation can be challenging when wearing personal protection equipment. LUS helped to assess the severity assessment for respiratory failure in Cases 3 and 4 as well as the recovery phase. In addition, LUS was a useful alternative to chest CT in children to whom radiation exposure can cause harm and in those who need sedation to perform chest CT [12,13]. Previous studies have stressed the accuracy and the diagnostic value of chest CT in managing patients with COVID-19 $[3,14,15]$. This case series illustrates the accuracy and the diagnostic value of LUS in managing patients with COVID or asymptomatic SARS-CoV-2 infection.

The goal of LUS in intensive care medicine and emergency medicine is to detect and manage the pathophysiological changes of cardiopulmonary diseases with an emphasis on point-of-care, rather than simply as a tool to reveal underlying anatomical abnormalities [7,1416]. While recent studies have emphasised the accuracy and the diagnostic value of chest CT in patients with COVID-19 $[3,17,18]$, LUS may be a good alternative test for CT scan, and it carries less risk of breaking airborne isolation and transmission of SARS-CoV-2 infection to others. Currently, a CT scan had been difficult because of keeping a COVID-19 patient in the negatively pressured mobile bed from the quarantine area to the examination room. Besides, more health care workers should accompany critically ill COVID-19 patients for their safety, which inevitably increases the risk of exposure to the virus during transportation. Also, in this study LUS was more sensitive than CXR and sometimes detected B-lines, indicative of subpleural lesions despite the absence of obvious changes on CXR. In an attempt to quantify the LUS findings, we adopted the machine's 


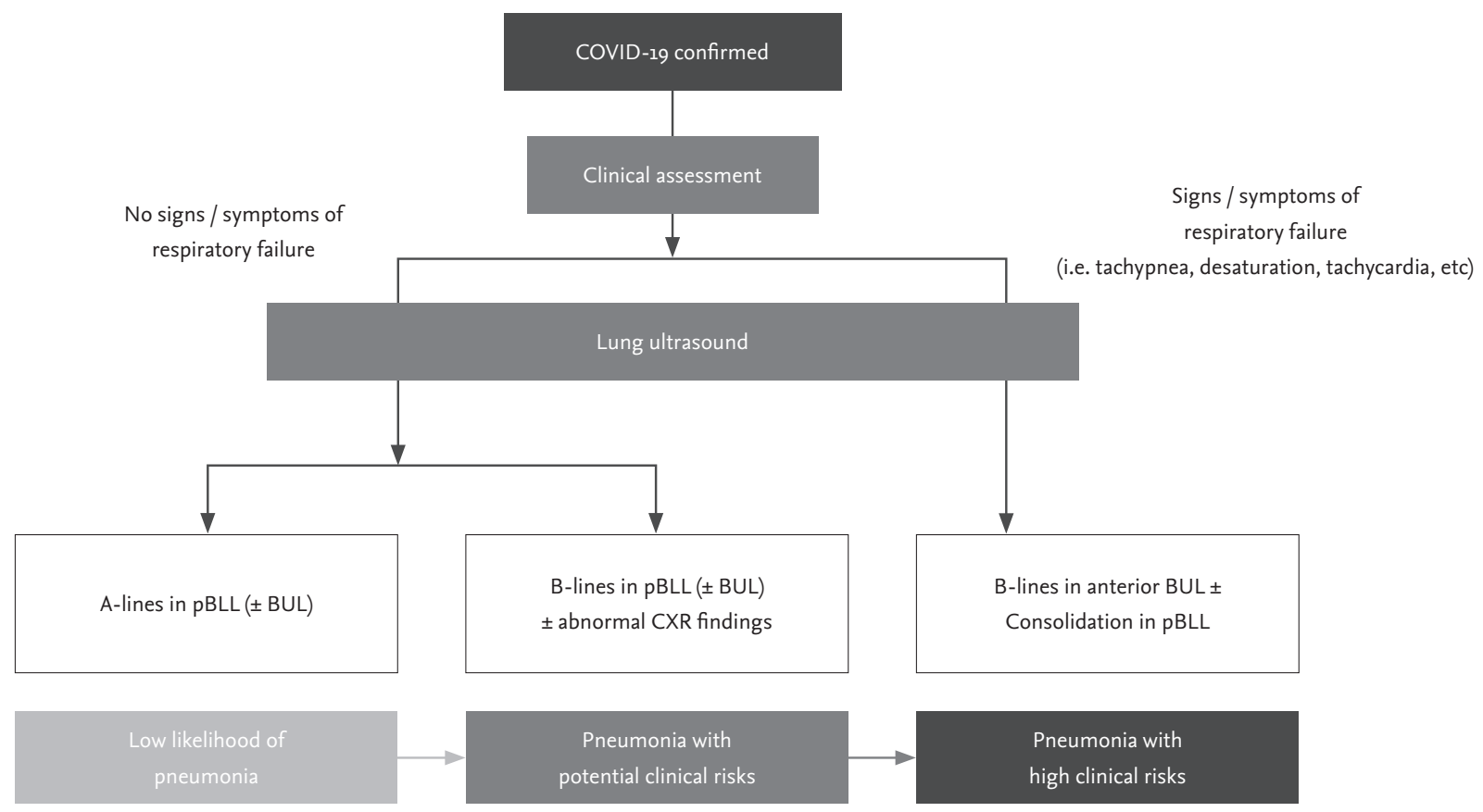

Figure 7. Proposed lung ultrasound screening algorithm for deciding on the management of patients with coronavirus disease 2019 (COVID-19). pBLL, posterior both lower lobe areas; BUL, both upper lobe areas; CXR, chest X-ray.

built-in algorithm of analysing characteristics of lesions, focused on interpreting B-lines and the lung aeration score. The automated quantification process helped to overcome the limitations of LUS, particularly its intra-operative variability and patient-related factors such as obesity and position $[19,20]$.

In the first adult and the first child patients (Cases 1 and 5), the LUS findings of improvement or deterioration during the period in which the patient was under observation agreed with the CT findings and with changes in the SARS-CoV-2 RT-PCR cycle threshold (Ct) values for RNA-dependent RNA polymerase ( $R d R p)$ gene (Supplementary Fig. 8A and 8B). This suggests that LUS might be a very useful tool for monitoring disease progression in COVID-19 patients [21].

Currently in South Korea, many asymptomatic or mildly symptomatic individuals are confirmed as having SARS-CoV-2 infection due to extensive use of RTPCR testing and thorough epidemiologic investigation of close contacts of individuals with confirmed infection. Due to outbreaks among members of religious organisations and inmates of psychiatric institutions in certain geographic areas including the city of Daegu and
Gyeongbuk Province, the number of individuals with confirmed infection overwhelmed the medical system, which limited the ability of medical staff to assess the severity of the COVID-19 in some patients during the first few weeks of the epidemic. As in other countries, there were many early deaths due to rapidly progressive disease in those regions. In such situations, using LUS for early detection of pulmonary involvement in patients with COVID-19 could be lifesaving, because it is very difficult to recognise patients developing acute respiratory failure with hypoxia in advance, especially within 48 hours after hospitalization. There is an urgent need for a rapid, reliable, and low-risk everyday pointof-care clinical tool that can be used to triage patients with SARS-CoV-2 infection and identify those at risk of respiratory failure $[4,22,23]$. As ultrasound examinations in the intensive care unit have been covered by national insurance in South Korea, it is also affordable especially for critically ill patients such as severe COVID-19. We propose the use of an LUS screening protocol as shown in Fig. 7.

There are some limitations to this study. First, we performed the chest CT prior to LUS for the index pa- 
tient, who did not have significant symptoms suggesting lower respiratory tract pathology. Therefore, there is a possibility that lesions cannot be detected if LUS was performed without knowing the CT results, given that the initial findings for COVID-19 are only focal GGO lesions. In other cases of acute respiratory distress syndrome, however, LUS could identify B-lines, ultrasound evidence of peripherally located GGO, or infiltrations on COVID-19 patients that corresponded to initial symptoms, even before the CXR. We believe that that LUS could be performed for confirmed COVID-19 patients without obvious lower respiratory tract symptoms, to guide very early clinical decisions.

Second, the radiographic findings obtained by CT scan and LUS could be at different stage of COVID-19 progress, as there was a time difference between those two measures. There was a time difference between CT scans and LUS from a few hours up to 2 days, depending on the hospital logistics and patients' condition, as well as the call schedule of the operator. However, with serial CXRs performed during the gap period, obvious changes were able to be recorded, and clinically estimated to some extent.

Third, the study was conducted by one ultrasound examiner and was a case series of six patients. As mentioned, inter-operative or even intra-operative variations can be of concern with LUS, and we were not able to address or calculate inter-operative variations with current study. However, with using built-in machine learning algorism for image standardisation and processing, we were able to quantify and characterise B-lines for all six case patients. Given the pandemic outbreaks occurring around the world, we hope the insufficient number of this research subjects could be overcome by subsequent large-scale studies. Especially, we hope the methodologies of longitudinal follow-up LUS on COVID-19 patients could be adopted by other researchers and clinicians, to reduce the risk of breaking isolations as well as radiation exposures. This could be especially valuable in children, who need mostly sedation, where it is difficult to repeat chest CT.

In conclusion, LUS was feasible and useful for a rapid, sensitive, affordable point-of-care screening tool to detect pneumonia without radiation hazard and suggest the severity of respiratory failure for COVID-19 patients.

\section{KEY MESSAGE}

1. Lung ultrasound is feasible and useful for screening tool to detect pneumonia in early phase without radiation hazard.

2. The disease severity assessed by lung ultrasound can help clinical decision making in severe coronavirus disease 2019 patients with respiratory failure.

\section{Conflict of interest}

No potential conflict of interest relevant to this article was reported.

\section{Acknowledgments}

We would like to thank Editage (www.editage.co.kr) for English language editing.

\section{REFERENCES}

1. Wu Z, McGoogan JM. Characteristics of and important lessons from the coronavirus disease 2019 (COVID-19) outbreak in China: summary of a report of 72314 cases from the Chinese Center for Disease Control and Prevention. JAMA 2020 Feb 24 [Epub]. https://doi.org/10.1001/ jama.2020.2648.

2. Li Y, Xia L. Coronavirus disease 2019 (COVID-19): role of chest CT in diagnosis and management. AJR Am J Roentgenol 2020;214:1280-1286.

3. Ai T, Yang Z, Hou H, et al. Correlation of chest CT and RT-PCR testing in coronavirus disease 2019 (COVID-19) in China: a report of 1014 cases. Radiology 2020 Feb 26 [Epub]. https://doi.org/10.1148/radiol.2020200642.

4. Buonsenso D, Pata D, Chiaretti A. COVID-19 outbreak: less stethoscope, more ultrasound. Lancet Respir Med 2020;8:e27.

5. Fang Y, Zhang H, Xie J, et al. Sensitivity of chest CT for COVID-19: comparison to RT-PCR. Radiology $2020 \mathrm{Feb}$ 19 [Epub]. https://doi.org/10.1148/radiol.2020200432.

6. Mojoli F, Bouhemad B, Mongodi S, Lichtenstein D. Lung ultrasound for critically ill patients. Am J Respir Crit Care Med 2019;199:701-714.

7. Volpicelli G, Elbarbary M, Blaivas M, et al. International evidence-based recommendations for point-of-care lung 
ultrasound. Intensive Care Med 2012;38:577-591.

8. Lichtenstein DA, Meziere GA. Relevance of lung ultrasound in the diagnosis of acute respiratory failure: the BLUE protocol. Chest 2008;134:117-125.

9. Rouby JJ, Arbelot C, Gao Y, et al. Training for lung ultrasound score measurement in critically ill patients. Am J Respir Crit Care Med 2018;198:398-401.

10. Short J, Acebes C, Rodriguez-de-Lema G, et al. Visual versus automatic ultrasound scoring of lung B-lines: reliability and consistency between systems. Med Ultrason 2019;21:45-49.

11. Shokoohi H, LeSaux MA, Roohani YH, Liteplo A, Huang C, Blaivas M. Enhanced point-of-care ultrasound applications by integrating automated feature-learning systems using deep learning. J Ultrasound Med 2019;38:1887-1897.

12. Copetti R, Cattarossi L. Ultrasound diagnosis of pneumonia in children. Radiol Med 2008;113:190-198.

13. Ho MC, Ker CR, Hsu JH, Wu JR, Dai ZK, Chen IC. Usefulness of lung ultrasound in the diagnosis of community-acquired pneumonia in children. Pediatr Neonatol 2015;56:40-45.

14. Enghard P, Rademacher S, Nee J, et al. Simplified lung ultrasound protocol shows excellent prediction of extravascular lung water in ventilated intensive care patients. Crit Care 2015;19:36.

15. Volpicelli G, Lamorte A, Tullio M, et al. Point-of-care multiorgan ultrasonography for the evaluation of undifferentiated hypotension in the emergency department.
Intensive Care Med 2013;39:1290-1298.

16. Lui JK, Banauch GI. Diagnostic bedside ultrasonography for acute respiratory failure and severe hypoxemia in the medical intensive care unit: basics and comprehensive approaches. J Intensive Care Med 2017;32:355-372.

17. Zhao X, Liu B, Yu Y, et al. The characteristics and clinical value of chest CT images of novel coronavirus pneumonia. Clin Radiol 2020;75:335-340.

18. Zhou Y, Yang GD, Feng K, et al. Clinical features and chest CT findings of coronavirus disease 2019 in infants and young children. Zhongguo Dang Dai Er Ke Za Zhi 2020;22:215-220.

19. Chavez MA, Shams N, Ellington LE, et al. Lung ultrasound for the diagnosis of pneumonia in adults: a systematic review and meta-analysis. Respir Res 2014;15:50.

2o. Pereda MA, Chavez MA, Hooper-Miele CC, et al. Lung ultrasound for the diagnosis of pneumonia in children: a meta-analysis. Pediatrics 2015;135:714-22.

21. Yousef N, Vigo G, Shankar-Aguilera S, De Luca D. Semiquantitative ultrasound assessment of lung aeration correlates with lung tissue inflammation. Ultrasound Med Biol 2020;46:1258-1262.

22. Cheung JC, Ho LT, Cheng JV, Cham EYK, Lam KN. Staff safety during emergency airway management for COVID-19 in Hong Kong. Lancet Respir Med 2020;8:e19.

23. Pan F, Ye T, Sun P, et al. Time course of lung changes at chest CT during recovery from coronavirus disease 2019 (COVID-19). Radiology 2020;295:715-721. 


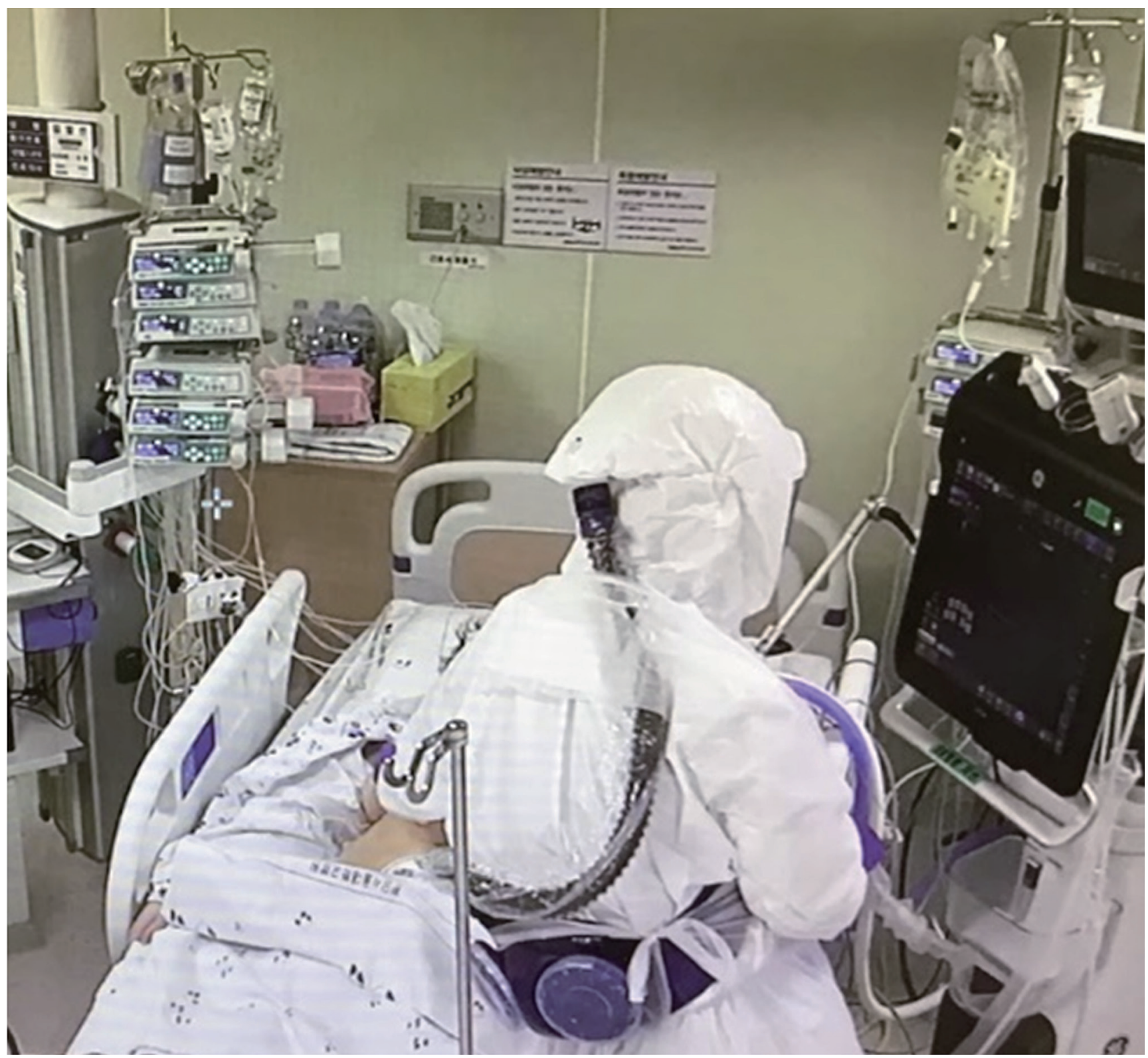

Supplementary Figure 1. Bedside lung ultrasound was done in an isolated, negative-pressure room for each patient by an independent physician with full personal protective equipment, including a powered air purifying respirator. 

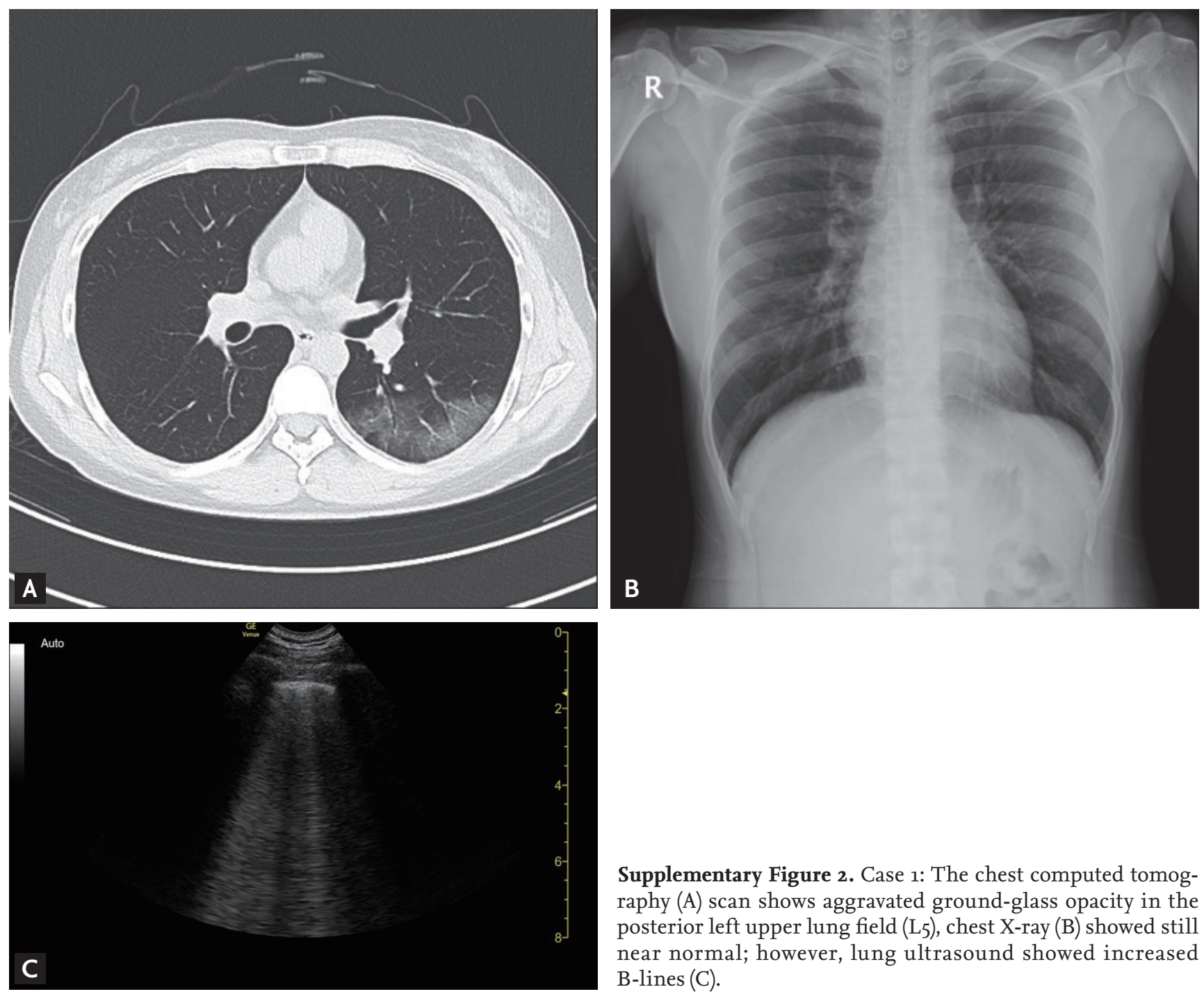

Supplementary Figure 2. Case 1: The chest computed tomography (A) scan shows aggravated ground-glass opacity in the posterior left upper lung field (L5), chest X-ray (B) showed still near normal; however, lung ultrasound showed increased B-lines (C). 


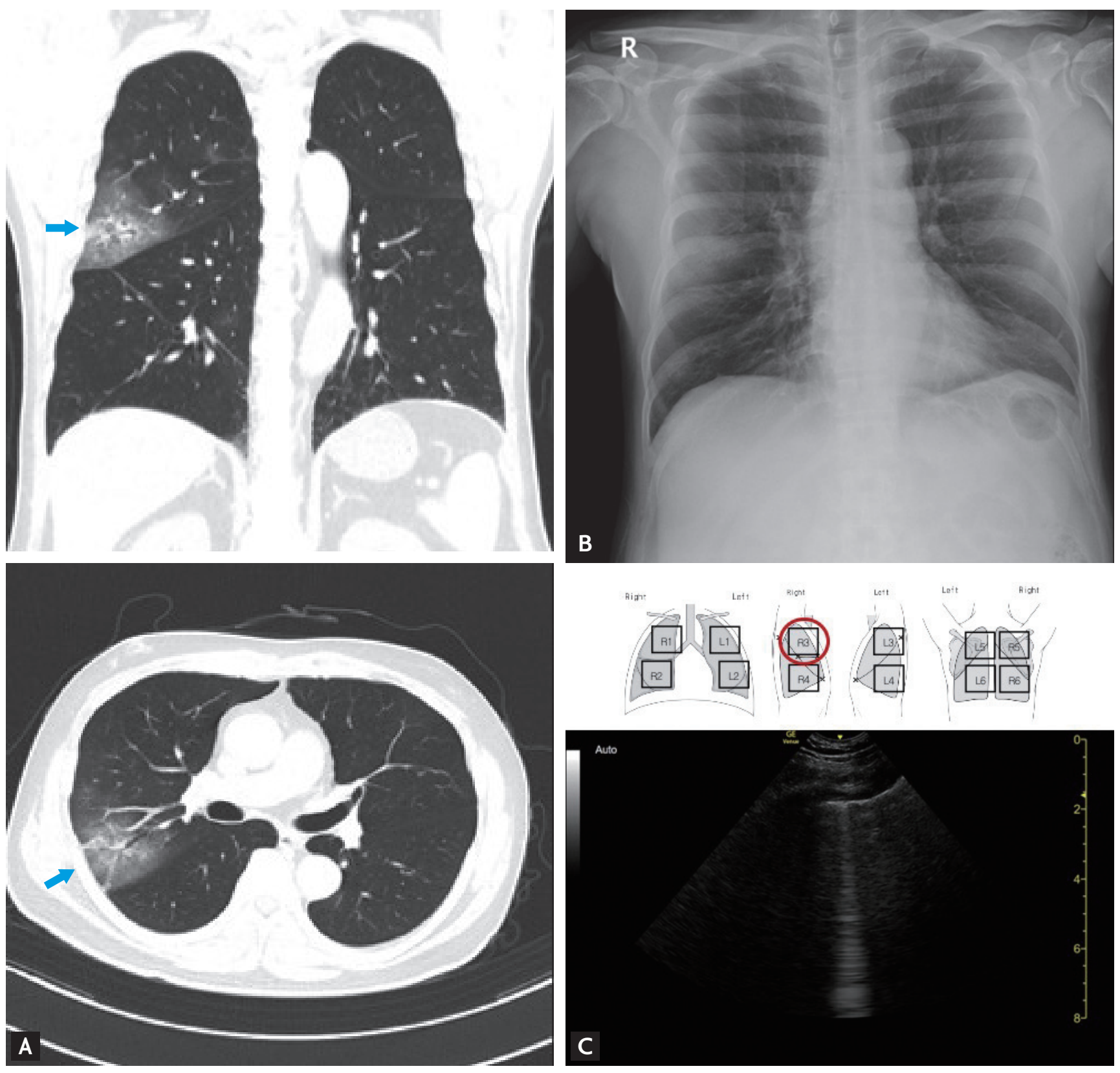

Supplementary Figure 3. Case 2: The chest computed tomography (A) scan shows ground-glass opacity (blue arrow) in the right upper lung field $\left(\mathrm{R}_{3}\right)$, chest X-ray (B) showed normal; however, lung ultrasound showed definite B-lines (C). 

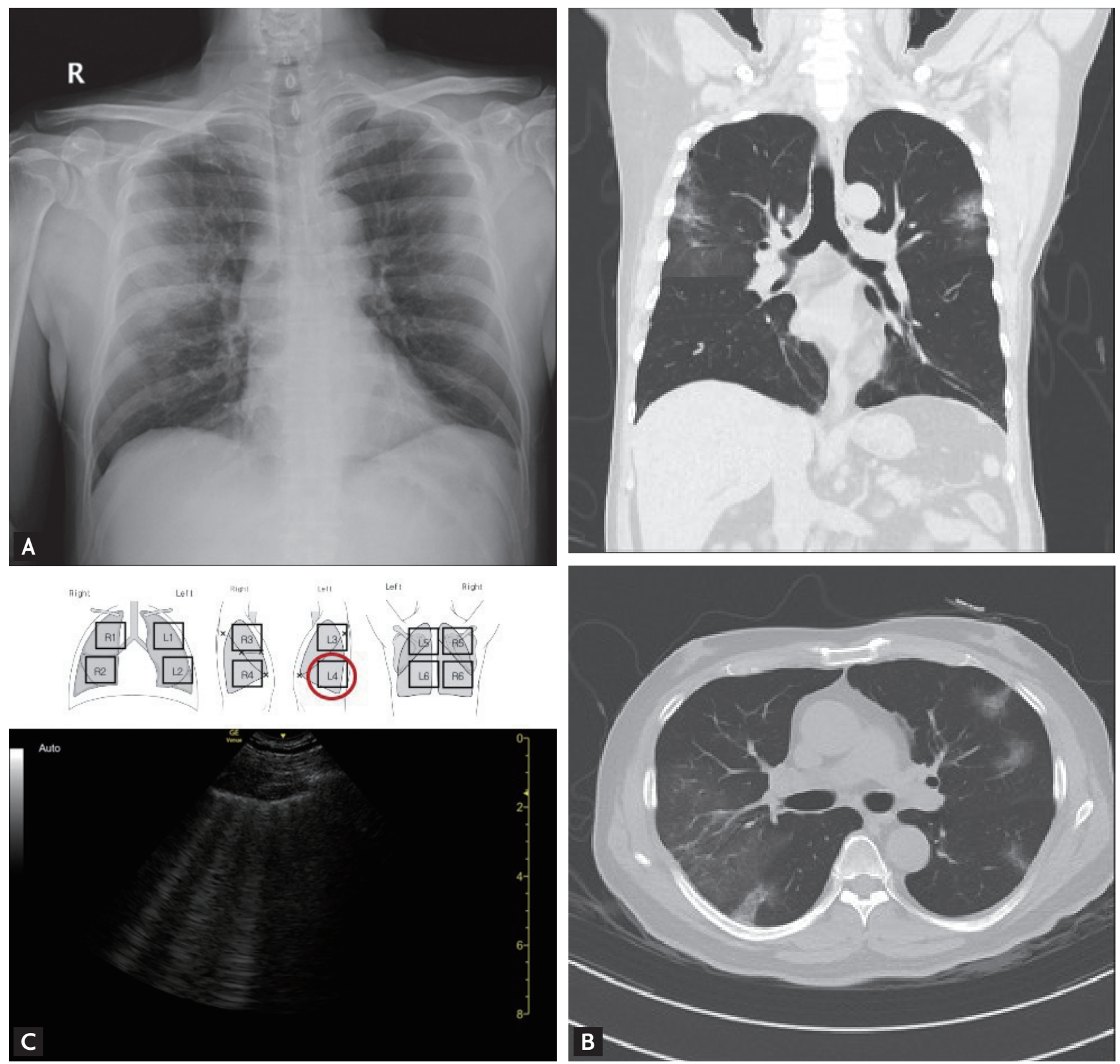

Supplementary Figure 4. Case 2: The chest X-ray shows subtle bilateral ground-glass opacities, which are more prominent in the right upper lung field (A), the chest computed tomography showed newly developed ground-glass opacities in the left lung fields (B); however, lung ultrasound detected B-lines in this area more earlier (C). 

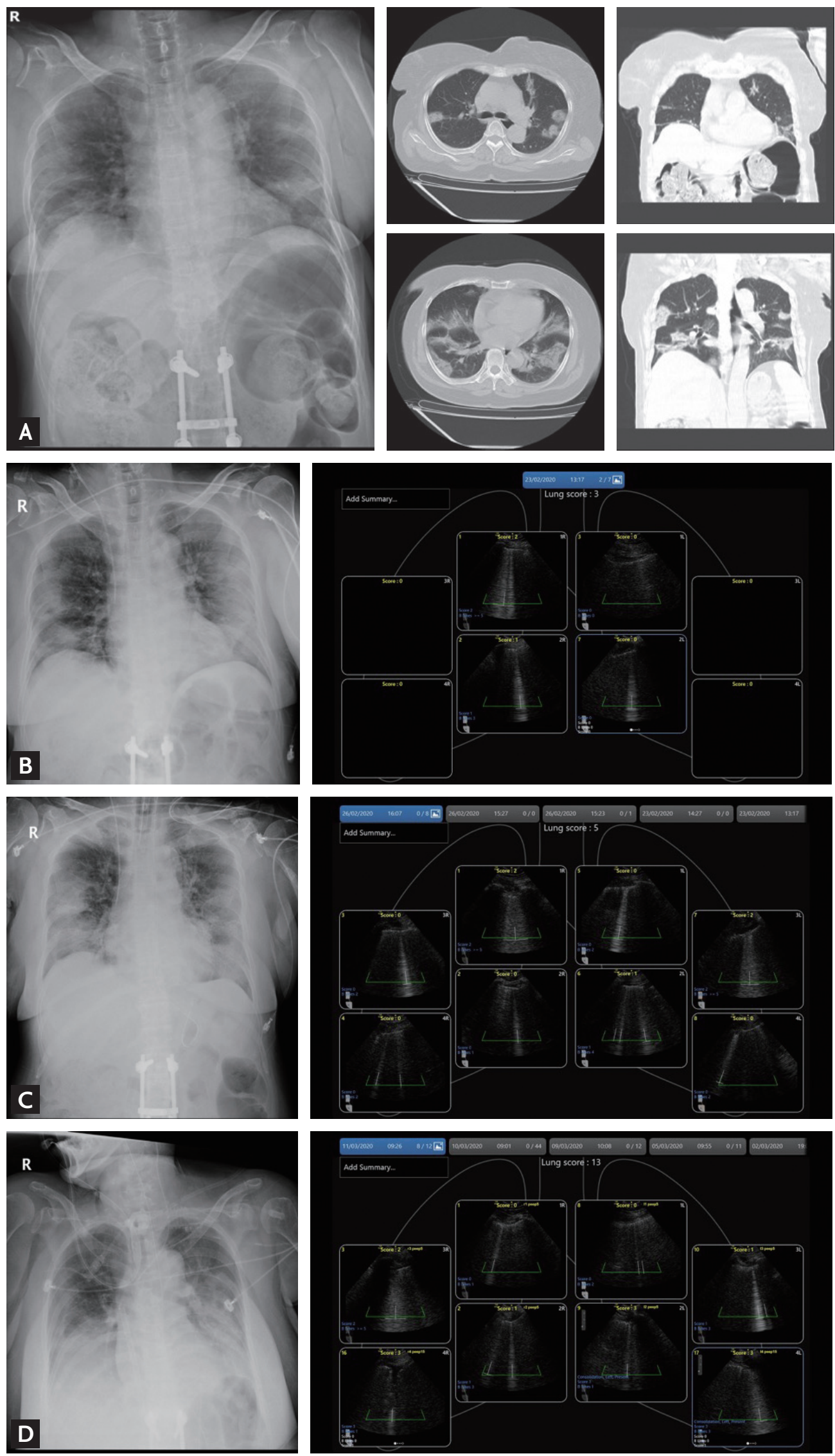

Supplementary Figure 5 . Case 4: The chest X-rays and computed tomography showing multi-focal ground-glass opacities in the lung fields bilaterally (A), lung ultrasound findings and aeration scores compared with chest $\mathrm{X}$-rays were changed with 3 (in anterior areas only) on admission day (B), 5 (in intubated state) on day $4(\mathrm{C})$, and 13 (in extracorporeal membrane assisted state) on day 18 (D). 

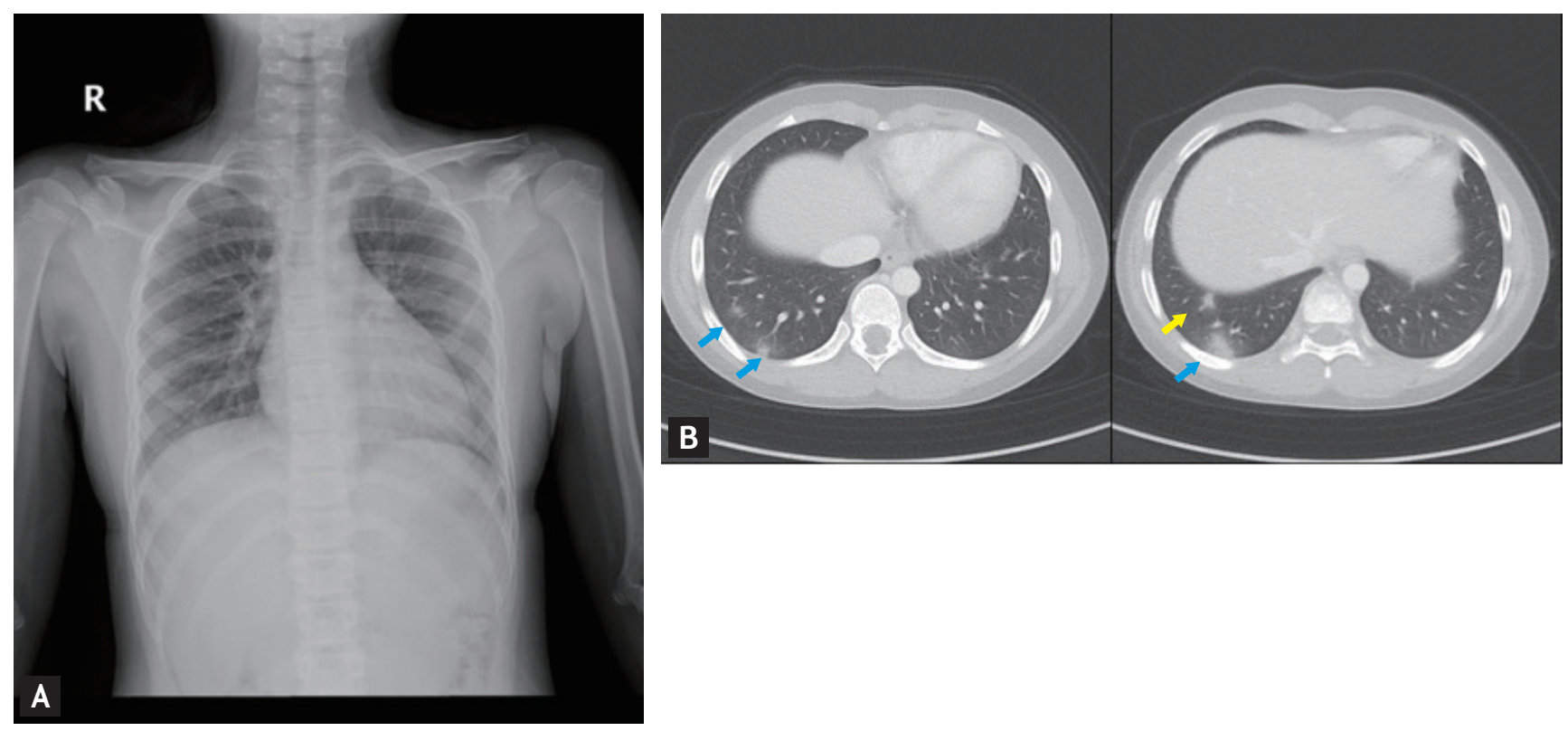

Supplementary Figure 6. Case 5: The chest X-ray showed no active lung lesions (A); however, chest computed tomography scan showed multi-focal ground-glass opacities (blue arrows) and consolidation (yellow arrow) in right lower lung field on Day 2 (B). 

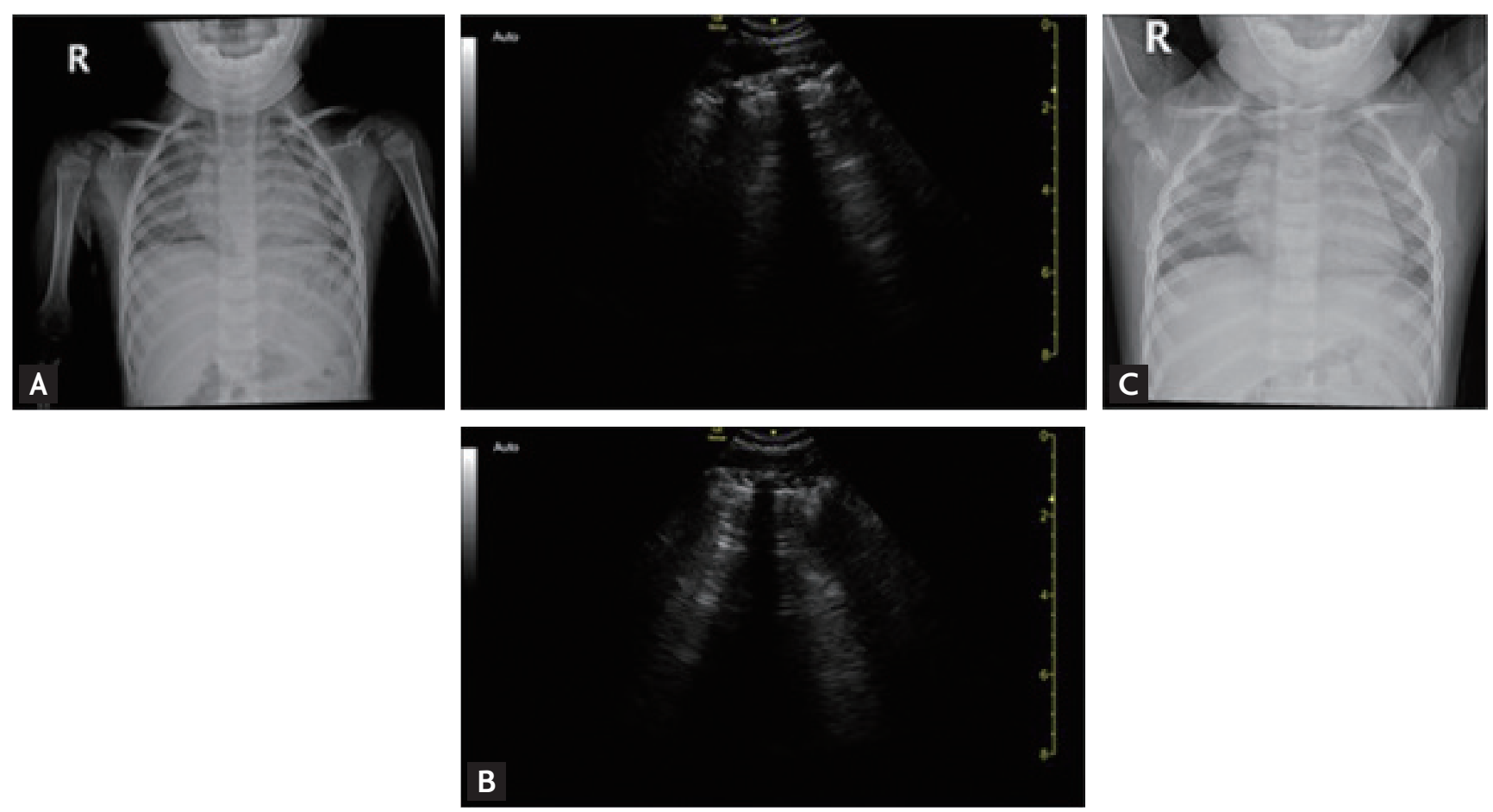

Day 2

Day 3

Day 4

Supplementary Figure 7. Case 6: Initial chest X-ray (A), lung ultrasound with both A-lines (B), and follow-up chest X-ray (C) showed all normal findings. 


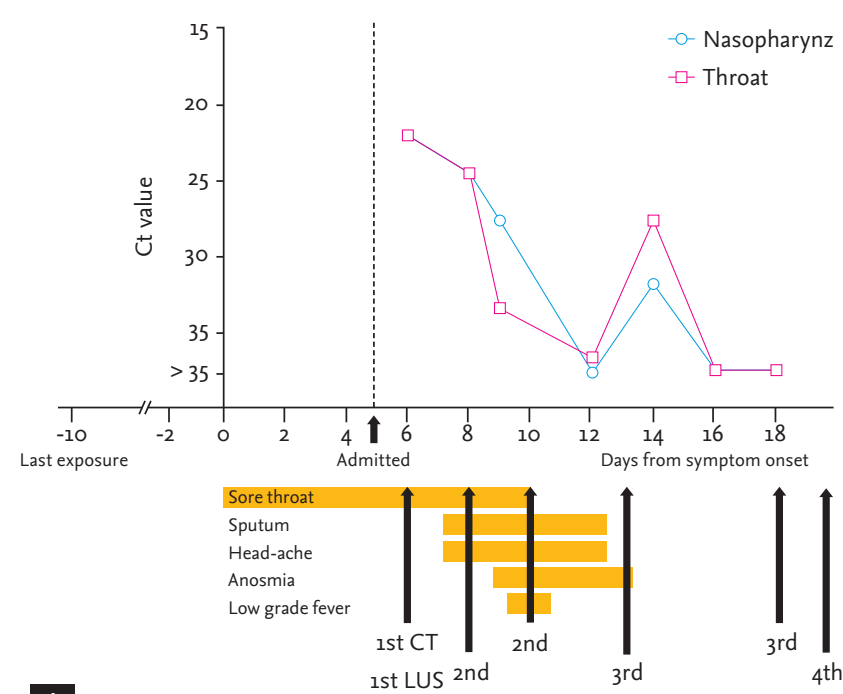

A

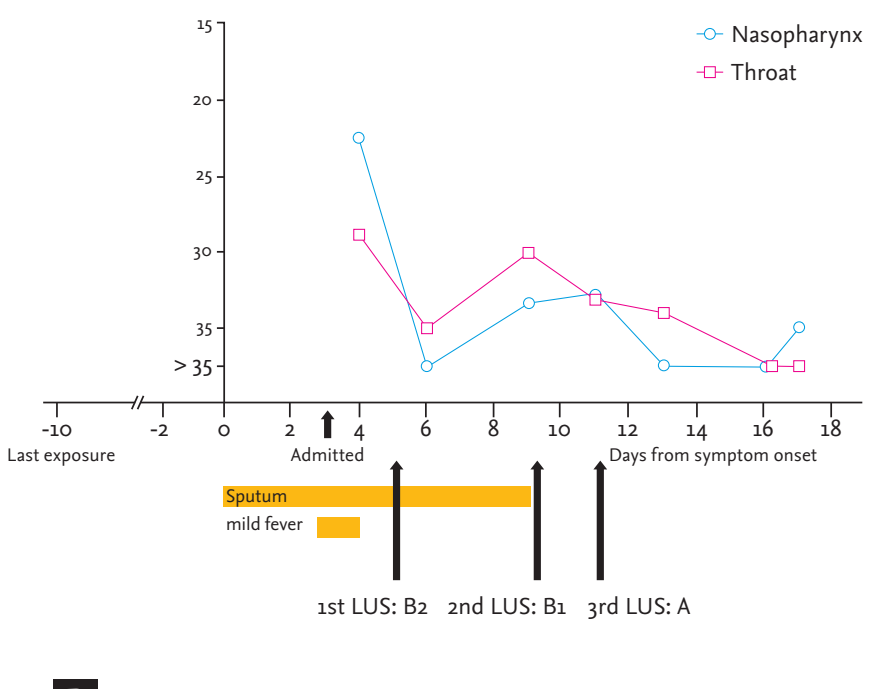

B

Supplementary Figure 8. Case 1 (A) and Case 5 (B): The temporal association between the lung ultrasound (LUS) findings, the symptoms, and severe acute respiratory syndrome coronavirus 2 reverse transcription-polymerase chain reaction cycle threshold (Ct) values for RdRp (RNA-dependent RNA polymerase) gene, according to the time since symptom onset. CT, computed tomography. 
Video 1. Case 1: Lung ultrasound showing typical B-lines in L5.

Video 2. Case 1: Lung ultrasound showing typical A-lines in R5.

Video 3. Case 1: Lung ultrasound showing subpleural consolidation (C) in R4.

Video 4. Case 5: The lung ultrasound showed improved lung aeration in R6; (A) B2-lines, (B) B1-lines, (C) A-lines. 
Cho YJ, et al. Lung ultrasound for COVID-19

Supplementary video 1. Case 1: Lung ultrasound showing increased B-lines compared with Video 1.

Supplementary video 2. Case 2: Lung ultrasound showing B-lines in R3.

Supplementary video 3. Case 2: Lung ultrasound showing B-lines in L4. This was detected before abnormal findings were shown in chest X-ray or computed tomography. 